\title{
Simultaneous Control of Frequency Fluctuation and Battery SOC in a Smart Grid using LFC and EV Controllers based on Optimal MIMO-MPC
}

\author{
Jonglak Pahasa ${ }^{\dagger}$ and Issarachai Ngamroo*
}

\begin{abstract}
This paper proposes a simultaneous control of frequency deviation and electric vehicles (EVs) battery state of charge (SOC) using load frequency control (LFC) and EV controllers. In order to provide both frequency stabilization and SOC schedule near optimal performance within the whole operating regions, a multiple-input multiple-output model predictive control (MIMO-MPC) is employed for the coordination of LFC and EV controllers. The MIMO-MPC is an effective modelbased prediction which calculates future control signals by an optimization of quadratic programming based on the plant model, past manipulate, measured disturbance, and control signals. By optimizing the input and output weights of the MIMO-MPC using particle swarm optimization (PSO), the optimal MIMO-MPC for simultaneous control of the LFC and EVs, is able to stabilize the frequency fluctuation and maintain the desired battery SOC at the certain time, effectively. Simulation study in a two-area interconnected power system with wind farms shows the effectiveness of the proposed MIMO-MPC over the proportional integral (PI) controller and the decentralized vehicle to grid control (DVC) controller.
\end{abstract}

Keywords: Load frequency control, Electric vehicle, State of charge, Model predictive control, Particle swarm optimization

\section{Introduction}

Electric vehicles (EVs) are significantly expected to be installed in the customer side [1-3]. Effective integrations of EVs into the grid are able to improve the power system ancillary services such as demand-side management [4], distribution network planning [5-6], wide area stability enhancement [7], and alleviation of frequency fluctuation [2, 8-14]. The power charging control of EVs in order to alleviate the frequency fluctuation has been proposed in [2]. In addition, the bidirectional power controls or vehicle to grid $(\mathrm{V} 2 \mathrm{G})$ of EV for alleviation of frequency deviation in the interconnected power system with wind farms have been proposed in [8-11]. The bidirectional charging/ discharging of $\mathrm{V} 2 \mathrm{G}$ and state of charge (SOC) control for a microgrid frequency stabilization is proposed in [12]. Besides, load frequency control (LFC) is successfully applied for coordinating with EV control to stabilize frequency deviation [11, 13-14]. Effective EV control with LFC signal is able to improve frequency stabilization [13].

This paper focuses on the coordination control of LFC and EVs in order to produce the desired SOC and frequency deviation. The review of existing control methods related

$\dagger$ Corresponding Author: Department of Electrical Engineering, Schoo of Engineering, University of Phayao, Phayao 56000, Thailand. (jonglak.pa@up.ac.th)

* Department of Electrical Engineering, Faculty of Engineering, King Mongkut's Institute of Technology Ladkrabang, Bangkok 10520,

Thailand. (knissara@kmitl.ac.th)

Received: December 5, 2015 ; Accepted: January 14, 2017 to the proposed coordinated control of EVs and LFC for smart grid frequency stabilization and SOC control, can be classified into 2 groups i.e., (1) existing EV charging with desired SOC and frequency control, and (2) existing EV combined with LFC for frequency control.

\section{1) The existing EV charging with desired SOC and frequency control, mainly consists of three methods as follows}

1.1) EV smart charging (SC) [9]: The $\mathrm{SC}$ is the improved version of the droop control [8] in order to provide the higher SOC after participated in the smart grid for frequency control. The SC consists of frequency droop control and scheduled charging power. The scheduled charging duration can be estimated but the actual plug-in duration of EV cannot be defined. Therefore, it is uncertian whether or not charging demand will be met by the SC.

1.2) EV charging with frequency regulation (CFR) [10]: The CFR is proposed to meet charging demand and suppress frequency deviation at the same time. The CFR is mainly composed of frequency droop control and improved scheduled charging power. When charging demand is considered in the V2G control, the constant scheduled charging power can be simplified as a function of the desired SOC, charging duration, and rated capacity of the EV battery. Therefore, the plug-out time as well as the expected SOC are able to be defined by the EV customer in advance. In this work, the SOC is increased based on the 
charging power against the frequency deviation which is varied in the acceptable ranges. However, the expected SOC may not be obtained when the frequency deviation more fluctuates out of the acceptable ranges. Because, when the SOC fluctuates outside the acceptable ranges, the SOC may not be increased as defined in advance. In addition, the SOC deviation is not included in the charging/discharging strategies. The SOC deviation is the difference between the actual SOC and the expected SOC.

1.3) SOC control with frequency stabilization using multiple model predictive control (MMPC) [12]: Eleven sub-MPCs are employed with different ranges of control signal in order to produce the desired frequency deviation and ranges of acceptable SOC. The disadvantages of this work consist of (1) the time duration required to obtain the desired SOC cannot be defined, and (2) the model is designed for obtainning the acceptable SOC ranges so that an exactly desired SOC may not be defined.

\section{2) The existing $E V$ combined with $L F C$ for frequency control, mainly consists of three methods, as follows.}

2.1) LFC by use of EVs [13]: The LFC by use of a number of the EVs is a centralized control where the area requirement (AR) as the $\mathrm{LFC}$ signal is sent to the conventional power plants and the EVs according to the response speed and the controllable capacity. This work shows that the LFC signal can be used as a part of control signal of EVs for suppression of frequency deviation. However, the SOC has not been considered to be charged for the higher level.

2.2) EV charging with LFC [14]: The EV charging with LFC is the coordinated $\mathrm{V} 2 \mathrm{G}$ control and conventional frequency controller for robust LFC. The battery SOC is controlled by the optimized SOC deviation control. This work shows that the effective coordinated control of LFC and $\mathrm{EV}$ is able to maintain frequency deviation and produce SOC, simultanuously. However, the time duration required to get the desired SOC cannot be defined.

2.3) Supplementary frequency regulation (SFR) with EVs [11]: The SFR with EVs is the improved version of the CFR [10] by bringing a large number of EVs into the centralized supplementary frequency control. The coordination between EVs and the power system control center is assigned as an aggregator. The aggregator calculates the total frequency regulation capacity (FRC) and expected V2G (EV2G) power. An EV customer increases the battery energy level for the next trip by the expected V2G power which is calculated based on the charging power among the frequency deviation. Here, the frequency deviation is varied in the acceptable ranges. Although, the expected V2G power of an EV for adjusting battery SOC level in this work is able to produce the expected SOC. However, the expected SOC may not be obtained when the frequency deviation more fluctuates out of the acceptable ranges as shown in [10]. Besides, the SOC deviation is not included in the charging/discharging strategies.

In order to improve the SOC schedule charging of an integrated EVs as well as to reduce the frequency deviation of the power system based on LFC and EV control signals, the multiple-input multiple-output model predictive control (MIMO-MPC) is employed for coordinating control of LFC and EVs bidirectional charging/discharging based on SOC schedule and frequency stabilization in this paper. The main advantages of the MPC are that the constraints may be explicitly specified into the problem formulation and the measured disturbances can be taken into consideration [15-18]. Constraints consist of ranges of possible MPC input-output due to manipulated variables, physical limitations, operating procedures or safety reasons, etc. Besides, measured disturbances such as wind power fluctuation, can be considered among MPC optimization of control signals. The applications of MPC for load frequency control have been successfully proposed in [19-20]. Besides, the MPC of bidirectional AC-DC converter for energy storage system has been proposed in [21].

In this paper, the MIMO-MPC is applied for frequency stabilization and SOC schedule control based on EV and LFC controllers. Multiple constraints such as the desired frequency deviation and battery SOC at the certain time are employed. Wind power fluctuation is defined as the measured disturbance. Besides, a particle swarm optimization (PSO) [22] is employed for searching the optimal MIMO-MPC input-output weights. Consequently, the optimal MIMO-MPC is able to reduce the system frequency deviation and obtain the desired battery SOC as setting in advance.

The organization of this paper is as follows. First, the study system and modeling of smart power system is described in section 2. Next, the MIMO-MPC controller design is provided in section 3. Subsequently, the experimental results are shown in section 4. Finally, the conclusion is provided.

\section{Study Power System and Modeling}

\subsection{Power system}

The two-area interconnected power system with large wind power penetration as shown in Fig. 1 is employed as the study system [14]. Each area consists of the wind power (WT), thermal power plant (THP), EVs, and load. The capacities of WT, THP, EVs, and load, in area 1, are $7000 \mathrm{MW}, 13400 \mathrm{MW}, 800 \mathrm{MW}$ and $18800 \mathrm{MW}$, respectively, and in area 2 are $2000 \mathrm{MW}, 3800 \mathrm{MW}, 1100$ $\mathrm{MW}$, and $4100 \mathrm{MW}$, respectively. The base power used is $20 \mathrm{MW}$ [14]. In this system the MIMO-MPCs are applied for coordinating control of $\mathrm{EV}$ and $\mathrm{LFC}$ based on the schedule of EV battery SOC and the reduction of power system frequency deviation. 


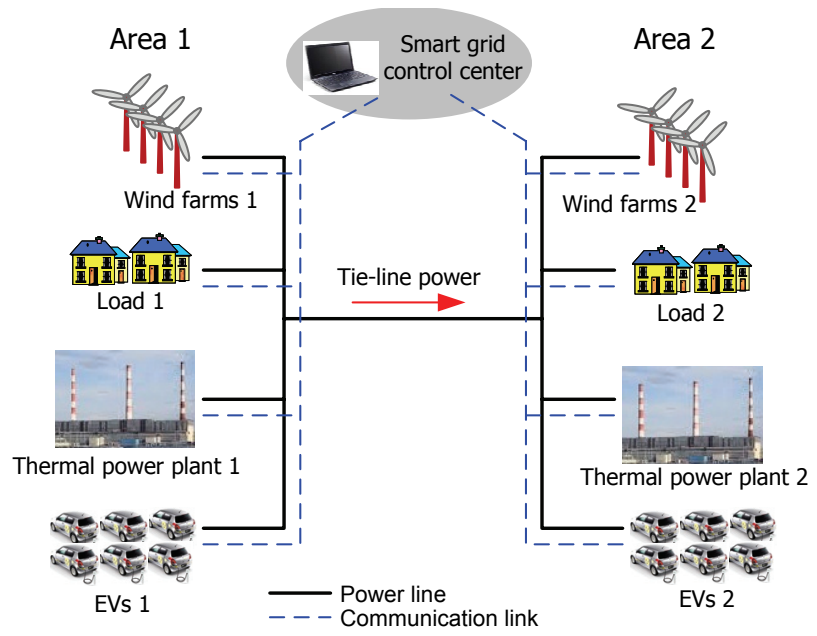

Fig. 1. Two-area interconnected power system

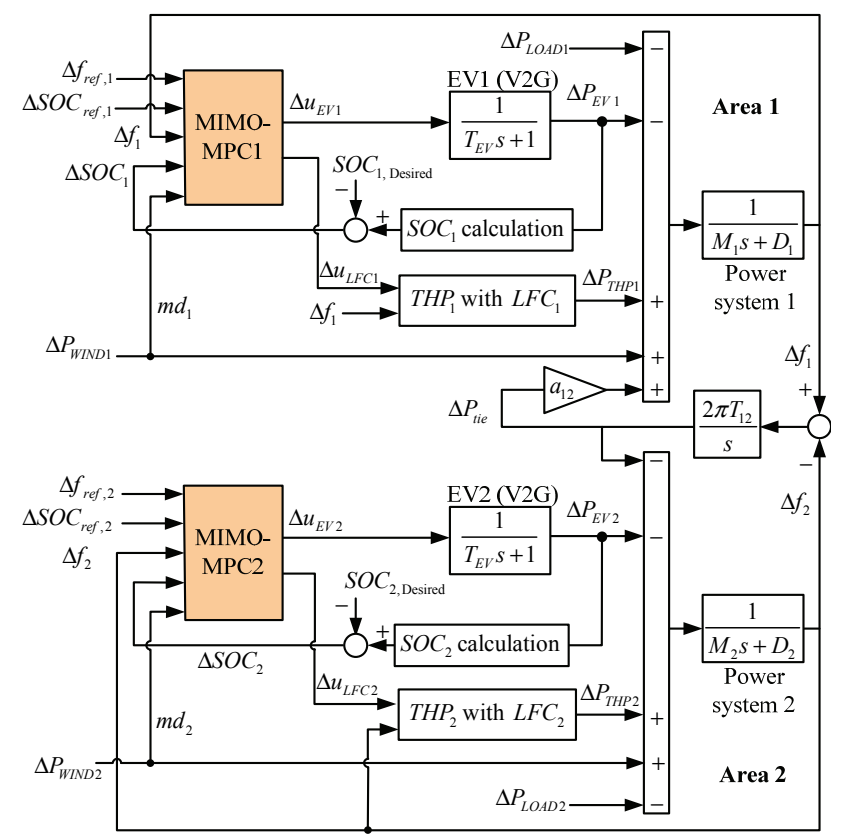

Fig. 2. Linearized model of the two-area interconnected power system

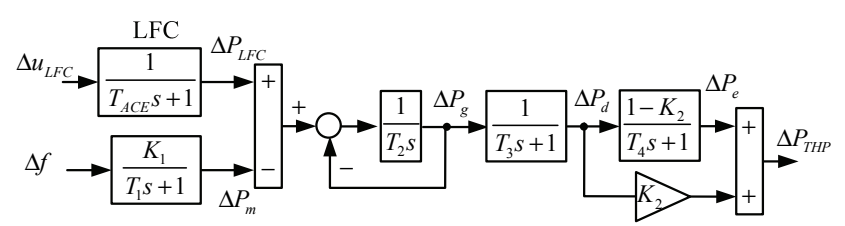

Fig. 3. Thermal power plant with LFC model

The linearized model of the two-area interconnected power system $[8,14,23]$ is shown in Fig. 2. In the EV model, the first-order transfer function with time delay $\left(T_{E V}\right)$ is employed [14]. The thermal generator with LFC is shown in Fig. 3. System parameters are given in Table 1 [8, 14, 23].

In order to handle the system non-linearity and the continuous variation in the operating point, a multiple-
Table 1. System parameters.

\begin{tabular}{c|c|c}
\hline Parameters & Area 1 & Area 2 \\
\hline Reference frequency, $f_{0}(\mathrm{~Hz})$ & 50 & 50 \\
\hline Inertia constant, $M(\mathrm{~s})$ & 9.02 & 9.02 \\
\hline Frequency characteristic of load except EV, & 2 & 2 \\
\hline ACE calculation time constant, $T_{A C E}(\mathrm{~s})$ & 10 & 10 \\
\hline Frequency bias factor, $K_{\text {system }}(\% \mathrm{MW} / \mathrm{Hz})$ & 10 & 10 \\
\hline Frequency characteristic per EV $(\mathrm{kW} / \mathrm{Hz})$ & 2.5 & 2.5 \\
\hline Number of EV & 110,000 & 150,000 \\
\hline Time constant of EV, $T_{E V}(\mathrm{~s})$ & 1 & 1 \\
\hline MW demand correction time constant, $T_{1}(\mathrm{~s})$ & 0.2 & 0.2 \\
\hline Control valve servomotor lag time constant, $T_{2}$ & 0.1 & 0.1 \\
\hline HP inlet piping lag time constant, $T_{3}(\mathrm{~s})$, & 0.25 & 0.25 \\
\hline Reheater, crossover and LP inlet piping lag time & 9 & 9 \\
\hline Fraction of power developed by HP, $K_{2}$ & 0.3 & 0.3 \\
\hline
\end{tabular}

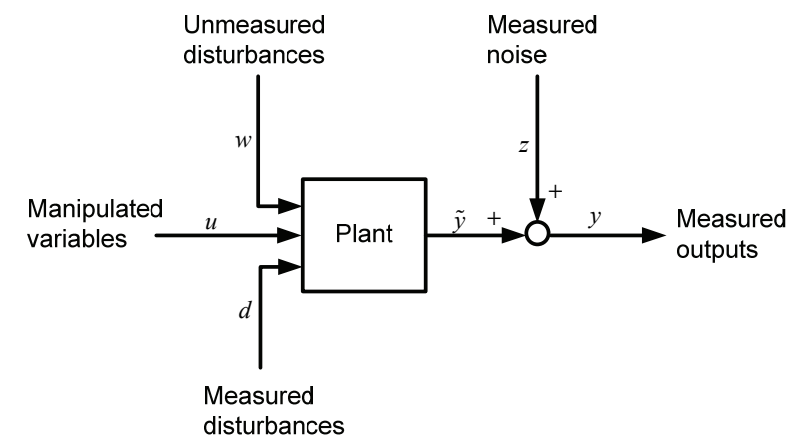

Fig. 4. State-space model for the MPC method

input multiple-output model predictive controller is suggested to provide near optimal performance within the whole operating regions.

\section{MIMO-MPC Controller Design}

In this paper, MIMO-MPC is applied for coordination control of LFC and EV controllers. The proposed MIMOMPC controller design has two objectives i.e., to reduce frequency deviation as well as to produce battery SOC level as expected in advance. The creative factor of the proposed algorithm is a synchronization generating of EV and LFC adaptive control signals using optimal MIMOMPC to produce the desired battery SOC and to improve the frequency stabilization, simultaneously.

\subsection{Overview of MPC}

The conventional MPC method is based on the current measurements and predictions of the future outputs [15-19]. The objective of the MPC is to determine a sequence of the control moves i.e., the manipulated input variable, so that the predicted response moves to the set point in an optimal manner. The state-space model for the MPC method [1819] can be represented as shown in Fig. 4. The general discrete-time linear time invariant (LTI) state-space equation can be expressed by [18-19], 


$$
\left.\begin{array}{rl}
x(k+1) & =A x(k)+B_{u} u(k)+B_{d} d(k)+B_{w} w(k) \\
y(k) & =\tilde{y}(k)+z(k) \\
& =C x(k)+D_{u} u(k)+D_{d} d(k)+D_{w} w(k)+z(k)
\end{array}\right\}
$$

where $x$ is the vector of $n$ state variables, $u$ is the $n_{u}$ manipulated variables, $d$ is the $n_{d}$ measured but freelyvarying inputs (i.e., the measured disturbances), $w$ is the $n_{w}$ unmeasured disturbances, $y$ and $\tilde{y}$ are the actual and predicted vectors of $n_{y}$ plant outputs, respectively, $n_{u}, n_{d}, n_{w}$, and $n_{y}$ are the numbers of manipulated variables, measured disturbances, unmeasured disturbances, and measured outputs, respectively, $z$ is the measured noise, $A$ is the state matrix of appropriate size, $B_{u}, B_{d}$ and $B_{w}$ are the relation of each state variable to the input signals, $C$ is the relation of the output signals to the state variables, $D_{u}, D_{d}$ and $D_{w}$, are the feed forward matrices that describe the relation of each output signal directly to the input signals. Subscripts $u, d$ and $w$ represent the manipulated variables, measured disturbances, and unmeasured disturbances, respectively. The variable $\tilde{y}(k)$ represents the plant output before the addition of measured noise.

\subsection{MIMO-MPC for LFC and SOC control}

The MIMO-MPC is the MPC for working with multipleinput multiple-output control system. The main challenge of the multiple-output MPC is to tune the controller to achieve multiple objectives. For example, if there are several outputs to be controlled, it might be necessary to prioritize so that the controller provides accurate setpoint tracking for the most important output, sacrificing others when necessary, e.g., when it encounters constraints [1819]. In this work, the MIMO-MPC is employed to produce LFC and EVs control signals using frequency and SOC deviations as the manipulated signals, and wind power as the measured disturbance.

The concept of MIMO-MPC for LFC and battery SOC coordinate control is shown in Fig. 5. Given that $u=\left[\Delta u_{L F C} \Delta u_{E V}\right], \tilde{u}=\left[\Delta \tilde{u}_{L F C} \Delta \tilde{u}_{E V}\right], y=[\Delta f \Delta S O C]$, and $\tilde{y}=[\Delta \tilde{f} \Delta \tilde{S} O C]$, where $\Delta u_{L F C}$ and $\Delta u_{E V}$ are the LFC and EV control signal deviations, respectively, $\Delta \tilde{u}_{L F C}$ and $\Delta \tilde{u}_{E V}$ are the predicted LFC and EV control signal deviations produced by MIMO-MPC, respectively, $\Delta f$ is the frequency deviation, $\triangle S O C$ is the battery SOC deviation (the difference between the actual SOC and the expected SOC), $\Delta \tilde{f}$ and $\triangle \tilde{S} O C$ are the predicted frequency and battery SOC deviations, respectively, the MPC method can be explained as follows.

At the current sampling instant, denoted by $k$, the MPC strategy calculates a set of $L$ values of the input $\{u(k+j-1), j=1,2,3, \ldots, L\}$. The set consists of the current input $u(k)$ and $L-1$ future inputs. The inputs are held constant after the $L$ control moves (Ref. to Fig. 5, position $k+L-1$ on the horizontal axis). The inputs are

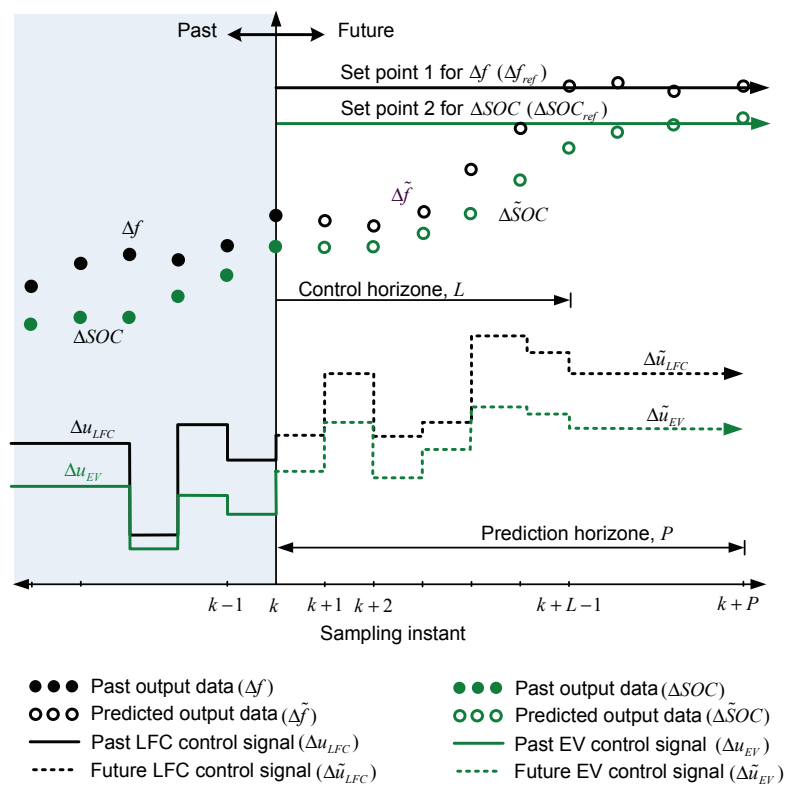

Fig. 5. Concept of the MIMO-MPC for LFC and SOC control

calculated, so that a set of $P$ predicted outputs $\{\tilde{y}(k+j), j=1,2, \ldots, P\}$ reaches the set point in an optimal manner.

The number of the predictions $P$ are referred to as the prediction horizon. The number of control moves $L$ are called the control horizon. Although a sequence of $L$ control moves are calculated at each sampling instant $(S)$, only the first move is actually implemented. Then a new sequence is calculated at the next sampling instant, after the new measurements become available again, only the first input move is implemented. This procedure is repeated at each sampling instant.

In this work, the MIMO-MPC is applied to the LFC of thermal generator and EV control, in order to stabilize the frequency fluctuation and obtain the battery SOC schedule, simultanuously. In addition, based on the charging need of user for the future journey and the various battery operating conditions during the $\mathrm{V} 2 \mathrm{G}$ mode, it is assumed that there is no EV control in each area during the first time of simulation. Then the $\mathrm{V} 2 \mathrm{G}$ is performed for charging the power of EVs during SOC which is less than the destination SOC ( $S O C<S O C_{\text {out }}$ ) for the next trip. Note that, among $\mathrm{V} 2 \mathrm{G}$ mode, the reduction of frequency deviation is performed and the $\mathrm{SOC}$ reaches the $S O C_{\text {out }}$ by the ramp rate function of time. The ramp rate $\mathrm{SOC}$ can be approximately defined by the real-time battery SOC variation provided by [9-11],

$$
S O C_{\mathrm{e}}(t)=S O C_{\mathrm{in}}+\left(\frac{S O C_{\text {out }}-S O C_{\mathrm{in}}}{T_{\text {out }}-T_{\text {in }}}\right) t, \quad T_{\text {in }} \leq t \leq T_{\text {out }}
$$

where $S O C_{\mathrm{e}}(t)$ is the expected SOC at time instant $t$, $S O C_{\text {in }}$ and $S O C_{\text {out }}$ are the initial SOC and destination 
SOC at plug-in and plug-out times of the EV battery, respectively, $T_{\text {in }}$ and $T_{\text {out }}$ are the plug-in and expected plug-out times for integrating $\mathrm{EV}$, respectively, and $t$ is the instant time. Although this work employs the linear SOC variation as the case study, the non-linear SOC pattern can be employed.

The control loop of the EV and LFC using MIMO-MPC controller are shown in Figs. 2 and 3. Here, the MIMOMPC1 and MIMO-MPC2 are designed for LFC and EV controllers in areas 1 and 2, respectively. Based on the overlapping decompositions method in [24], the state space equation of the power system area $i$ can be defined as,

$$
\begin{aligned}
& \dot{x}_{i}=A_{i} \cdot x_{i}+B_{i} \cdot u_{i} \\
& y_{i}=C_{i} \cdot x_{i}+D_{i} \cdot u_{i} \\
& A_{i}=\left[\begin{array}{ccccccccc}
\frac{-D_{i}}{M_{i}} & 0 & 0 & 0 & 0 & \frac{K_{2, i}}{M_{i}} & \frac{1}{M_{i}} & \frac{-K_{3, i}}{M_{i}} & \frac{a_{12}}{M_{i}} \\
0 & 0 & 0 & 0 & 0 & 0 & 0 & K_{4, i} & 0 \\
0 & 0 & \frac{-1}{T_{A C E, i}} & 0 & 0 & 0 & 0 & 0 & 0 \\
\frac{K_{1, i}}{T_{1, i}} & 0 & 0 & \frac{-1}{T_{1, i}} & 0 & 0 & 0 & 0 & 0 \\
0 & 0 & \frac{1}{T_{2, i}} & \frac{-1}{T_{2, i}} \frac{-1}{T_{2, i}} & 0 & 0 & 0 & 0 \\
0 & 0 & 0 & 0 & \frac{1}{T_{3, i}} & \frac{-1}{T_{3, i}} & 0 & 0 & 0 \\
0 & 0 & 0 & 0 & 0 & \frac{\left(1-K_{2, i}\right)}{T_{4, i}} & \frac{-1}{T_{4, i}} & 0 & 0 \\
0 & 0 & 0 & 0 & 0 & 0 & 0 & \frac{-1}{T_{E V, i}} & 0 \\
2 \pi T_{i j} & 0 & 0 & 0 & 0 & 0 & 0 & 0 & 0
\end{array}\right] \\
& B_{i}=\left[\begin{array}{cc}
0 & 0 \\
0 & 0 \\
\frac{1}{T_{A C E, i}} & 0 \\
0 & 0 \\
0 & 0 \\
0 & 0 \\
0 & 0 \\
0 & \frac{1}{T_{E V, i}} \\
0 & 0
\end{array}\right], C_{i}=\left[\begin{array}{lllllllll}
1 & 0 & 0 & 0 & 0 & 0 & 0 & 0 & 0 \\
0 & 1 & 0 & 0 & 0 & 0 & 0 & 0 & 0
\end{array}\right],
\end{aligned}
$$

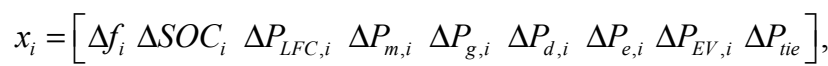

$$
\begin{aligned}
& u_{i}=\left[\begin{array}{ll}
\Delta u_{L F C, i} & \Delta u_{E V, i}
\end{array}\right], \quad y_{i}=\left[\begin{array}{ll}
\Delta f_{i} & \Delta S O C_{i}
\end{array}\right] \text {. }
\end{aligned}
$$

$$
\begin{aligned}
\min _{u(k) \in H} \sum_{j=1}^{H} & {\left[y_{i}(k+j)-r_{i}(k+j)\right]^{T} W_{y, i}\left[y_{i}(k+j)-r_{i}(k+j)\right] } \\
+ & {\left[u_{i}(k)-u_{i}(k-1)\right]^{T} W_{u, i}\left[u_{i}(k)-u_{i}(k-1)\right] }
\end{aligned}
$$

subject to $\Delta f_{i, \min } \leq \Delta f_{i} \leq \Delta f_{i, \max }$,

$$
\begin{aligned}
& \Delta S O C_{i, \min } \leq \Delta S O C_{i} \leq \Delta S O C_{i, \max }, \\
& \Delta u_{L F C, i, \min } \leq \Delta u_{L F C, i} \leq \Delta u_{L F C, i, \max }, \\
& \Delta u_{E V, i, \min } \leq \Delta u_{E V, i} \leq \Delta u_{E V, i, \max },
\end{aligned}
$$

where $r_{i}=\left[\Delta f_{r e f, i} \Delta S O C_{r e f, i}\right]$ is the reference set point, $u_{i}=\left[\Delta u_{L F C, i} \Delta u_{E V, i}\right]$ is the control signal, $\Delta S O C_{i}=$ $S O C_{\mathrm{e}, i}-S O C_{i}$, is the deviation of the actual SOC from the expected SOC, $\Delta f_{i, \min }$ and $\Delta f_{i, \max }$ are the minimum and maximum frequency deviations, respectively, $\Delta S O C_{i, \min }$ and $\Delta S O C_{i, \max }$ are the minimum and maximum SOC deviations, respectively, $\Delta u_{L F C, i, \text { min }}$ and $\Delta u_{L F C, i, \max }$ are the minimum and maximum LFC control signal deviations, respectively, $\Delta u_{E V, i, \min }$ and $\Delta u_{E V, i, \max }$ are the minimum and maximum EV control signal deviations, respectively, $W_{y, i}$ and $W_{u, i}$ are the positive semidefinite weighting matrices for the input and control signals, respectively, and $L$ is the control horizon.

\subsection{Detail strategy of the MIMO-MPC for LFC and SOC control}

Detail of the MIMO-MPC for LFC and SOC control is shown in Fig. 6. The main components of the MIMO-MPC controller are the prediction model bank, the optimization problem (MPC) and the state estimator bank [26]. More details strategy can be explained as follows.

Step 1: Define the "Model bank" of the power system area $i$, with LFC (Thermal power plant) and EV, as in (3) and (4). The study system is linearized around normal operating point, so that the $A_{i}, B_{i}$ and $C_{i}$ can be defined.

Step 2: At the current sampling time instant defined as $k$, the "Estimator bank" estimate the plant state $\tilde{x}^{n}(k \mid k)$ and the disturbance state $\tilde{x}_{d}^{n}(k \mid k)$. Then the "MPC" compute the optimal set of input $\left\{\left[\Delta u_{L F C}(k+n-1)\right.\right.$, $\left.\left.\Delta u_{E V}(k+n-1)\right], n=1,2,3, \ldots, L\right\}$ to produce the optimal output $\{[\Delta \tilde{f}(k+n), \Delta \tilde{S} O C(k+n)], n=1,2, \ldots, P\}$ using the MIMO-MPC optimization problem as in (5). The optimal output should be located close to the set point or the target
Where $\Delta f_{i}$ is the changes of frequency, $\triangle S O C_{i}$ is the changes of SOC, $\Delta P_{L F C}$ is the changes of output power of LFC, $\Delta P_{m, i}, \Delta P_{g, i}, \Delta P_{d, i}$ and $\Delta P_{e, i}$ are the changes of output power of MW demand correction droop control, servomotor valve control, HP inlet piping, and reheater of thermal power plant, respectively, $\Delta P_{E V, i}$ is the changes of EV power, $\Delta u_{L F C, i}$ and $\Delta u_{E V, i}$ are the changes of control 


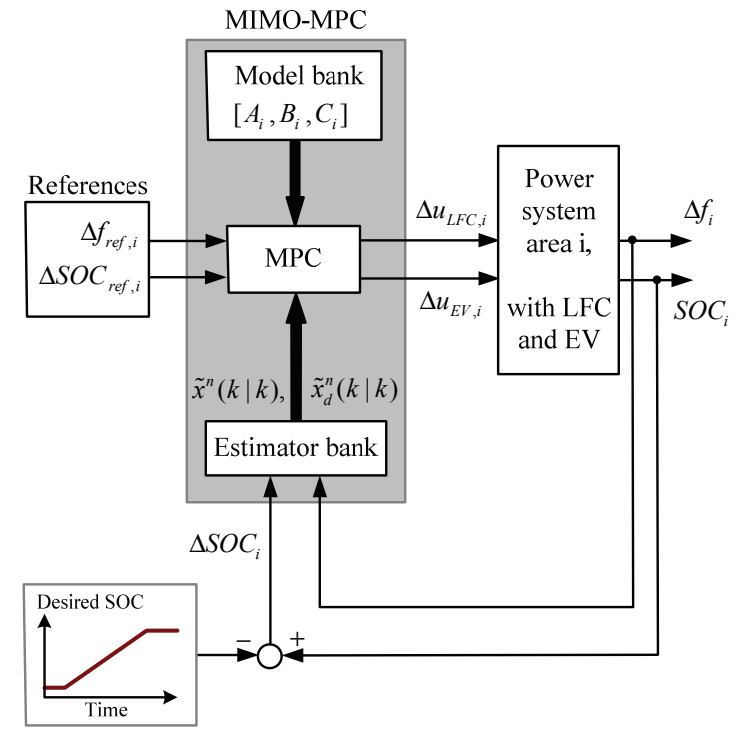

Fig. 6. Detail of the MIMO-MPC for LFC and battery SOC control

of the control action $\left[\Delta f_{\text {ref }}, \Delta S O C_{\text {ref }}\right]$.

Step 3: Use the $1^{\text {st }}$ predicted control signals i.e. $\left[\Delta u_{L F C}(k), \Delta u_{E V}(k)\right]$ as the actual input control signals for the LFC and EV controllers, respectively.

Step 4: $k=k+1$, return to step 2 .

\subsection{MIMO-MPC weight tuning}

The MPC weights ( $W_{y}$ and $W_{u}$ ) for LFC and EV controllers are simultanuously tunned by a particle swarm optimization (PSO) [22]. The objective function of the optimization is defined based on the integral absolute error $(I A E)$ of the SOC and frequency deviations. Consequently, the objective function can be defined as,

Minimize $I A E_{i}=\int_{T_{\text {in }}}^{T_{\text {out }}}\left|\Delta S O C_{i}\right| d t+\int_{T_{\text {in }}}^{T_{\text {out }}}\left|\Delta f_{i}\right| d t$

Subject to $W_{y, n, i, \min } \leq W_{y, n, i} \leq W_{y, n, i, \max }$,

$$
W_{u, n, i, \min } \leq W_{u, n, i} \leq W_{u, n, i, \max }, i=1,2, \quad n=1,2
$$

where $W_{y, n, i}$ and $W_{u, n, i}$ are the $n^{\text {th }}$ input and output weights, $W_{y, n, i, \min }$ and $W_{y, n, i, \max }$ are the minimum and maximum $n^{\text {th }}$ input weights, respectively, $W_{u, n, i, \min }$ and $W_{u, n, i, \max }$ are the minimum and maximum $n^{\text {th }}$ output weights, respectively, of the MIMO-MPC at area $i, T_{i n}$ and $T_{\text {out }}$ are the plug-in and plug-out times, respectively. Here, $n=1$ and $n=2$ for the LFC and EV controllers, respectively.

\section{Simulation Results}

To validate the effectiveness of the proposed method,
Table 2. PI controller parameters

\begin{tabular}{c|c|c|c|c}
\hline \multirow{2}{*}{} & \multicolumn{2}{|c|}{ Area 1 } & \multicolumn{2}{c}{ Area 2 } \\
\cline { 2 - 5 } & $K_{p}$ & $K_{i}$ & $K_{p}$ & $K_{i}$ \\
\hline LFC & 0.6745 & 0.4132 & 0.6986 & 0.3283 \\
\hline EV & 0.8997 & 0.5703 & 0.6850 & 0.3672 \\
\hline
\end{tabular}

Table 3. SOC and time setting of case studies

\begin{tabular}{|c|c|c|c|c|c|}
\hline Details & Area & Case 1 & Case 2 & Case 3 & Case 4 \\
\hline \multirow{2}{*}{$\begin{array}{c}\text { SOC } \\
\text { initial }\end{array}$} & 1 & $30 \%$ & $20 \%$ & $50 \%$ & $20 \%$ \\
\hline & 2 & - & $20 \%$ & $50 \%$ & $20 \%$ \\
\hline \multirow{2}{*}{$\begin{array}{c}\text { SOC } \\
\text { destination }\end{array}$} & 1 & $70 \%$ & $80 \%$ & $80 \%$ & $80 \%$ \\
\hline & 2 & - & $80 \%$ & $80 \%$ & $80 \%$ \\
\hline \multirow{2}{*}{$\begin{array}{c}\text { Integrated } \\
\text { time }\end{array}$} & 1 & $8: 00-16: 00$ & $8: 00-16: 00$ & $8: 00-12: 00$ & $8: 00-16: 00$ \\
\hline & 2 & - & $8: 00-16: 00$ & $8: 00-12: 00$ & $8: 00-12: 00$ \\
\hline
\end{tabular}

time simulations of the study system have been carried out. In the design of MIMO-MPC, the parameters and ranges of constraints of MIMO-MPC are set as follows, $P=10, L=3, S=0.1 \mathrm{~s},\left[\Delta f_{\min } \Delta f_{\text {max }}\right]=\left[\begin{array}{ll}-0.05 & 0.05\end{array}\right]$ and

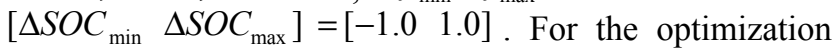
of MIMO-MPC weights, the ranges of search parameters for the PSO are set as follows; maximum particle velocity $=4$, population size $=24, w_{\min }=0.4, w_{\max }=0.9, c_{1}=2$, $c_{2}=2$, and maximum iteration $=150$, where $w_{\min }$ and $w_{\max }$ are the minimum and maximum weighting functions, respectively, $c_{1}$ and $c_{2}$ are the relative weights of the local and global best positions, respectively. The optimal weights are given as follows,

$$
\begin{aligned}
\text { MIMO-MPC1: } & W_{y, 1,1}=9.8083 \times 10^{-3}, W_{u, 1,1}=0.9744, \\
W_{y, 1,2} & =9.9229 \times 10^{-3}, W_{u, 1,2}=0.9851, \\
\text { MIMO-MPC2: } & W_{y, 2,1}=9.9568 \times 10^{-3}, W_{u, 2,1}=0.9533, \\
& W_{y, 2,2}=9.6539 \times 10^{-3}, W_{u, 2,2}=0.9914,
\end{aligned}
$$

where MIMO-MPC1 and MIMO-MPC2 are the MIMOMPC for areas 1 and 2, respectively.

In addition, the proposed MIMO-MPC is compared with the proportional integral (PI) controller and the decentralized V2G control (DVC) controller [10]. Details of the PI controllers of LFC and EV are provided in [14] and [12], respectively. Besides, the PI parameters are tuned by PSO [22]. The objective function of the PI optimization is defined based on the IAE same as the MIMO-MPC. As a result, the optimal PI controllers are obtained as shown in Table 2, where $K_{p}$ and $K_{i}$ are the proportional and integral gains of the PI controller, respectively. Besides, the DVC is designed based on the method in [10] with specification of frequency deviation $\pm 0.05 \mathrm{~Hz}$, same as the MIMO-MPC. Additionally, in order to compare with the proposed method, all EVs of each area are assumed to be started with the same time and the same SOC.

In the simulation studies, it is supposed that the study system is performed under the random load pattern and wind power as shown in Figs. 7 (a) and (b), respectively. 
The detailed models of random load pattern and wind power are provided in [25]. Here, wind power is produced by wind speed model with fixed blade pith angle in [25]. However, size of system in [25] is smaller than the system in this paper. In [25], one area (microgrid) system is employed. In this paper, two-area interconnected power system is used as the study system. Besides, the way to display case study data of this paper and [25] are different. Details are as follows,

1) For Fig. 7 (a), this paper shows the variation changes from mean value $(-0.02$ to $+0.02 \mathrm{pu})$. Reference [25] shows random load pattern in term of mean value $[0.78$ to 0.80 pu). Duration in [25] is 3 hour and duration in this work is 8 hour. When considering in data, they are not different.

2) For Fig. 7 (b), this paper shows wind power data while reference [25] shows wind speed. As explained in

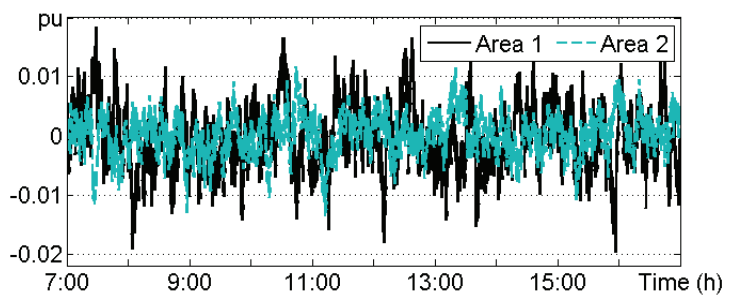

(a)

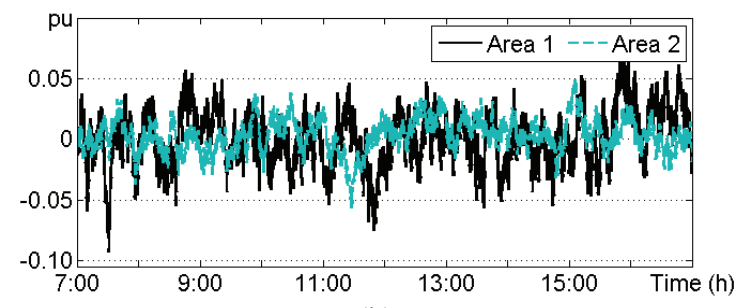

(b)

Fig. 7. Case studies: (a) random load pattern; (b) wind power

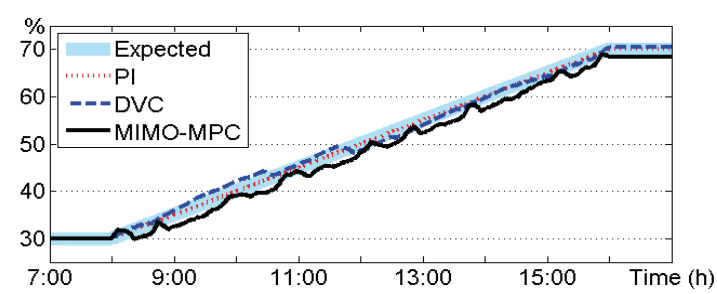

(a)

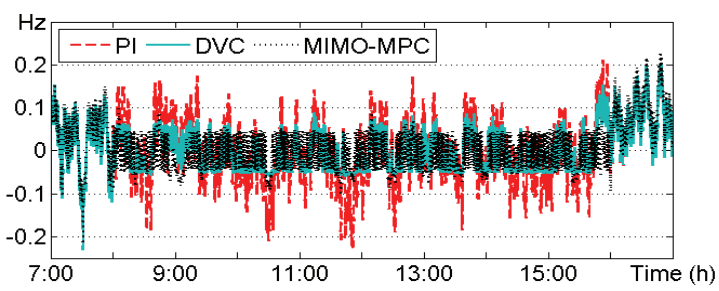

(b)

Fig. 8. Simulation results in case 1: (a) battery SOC; (b) frequency deviation
[25] wind speed is used to produce wind power by adjusting blade pitch angle.

Table 3 shows SOC and time setting of case studies. As explained in [10], EVs are integrated into area 1 (not into area 2). Therefore, for case 1 in this work, EVs are assumed to be integrated into area 1. Figs. 8 (a) and (b) show battery SOC and frequency deviation of case 1 , respectively. It can be seen that the DVC and MIMO-MPC are able to produce the desired battery SOC as well as reduce the frequency fluctuation, effectively. In contrast, the PI controller is able to produce battery SOC. But the system frequency deviation in case of PI controller is higher than other controllers.

For case 2, EVs are assumed to be integrated into both areas. Battery SOC of the PI, DVC, and MIMO-MPC controllers in areas 1 and 2, are shown in Figs. 9 (a) and (b),

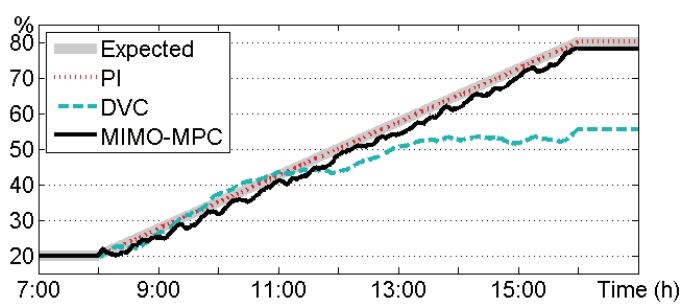

(a)

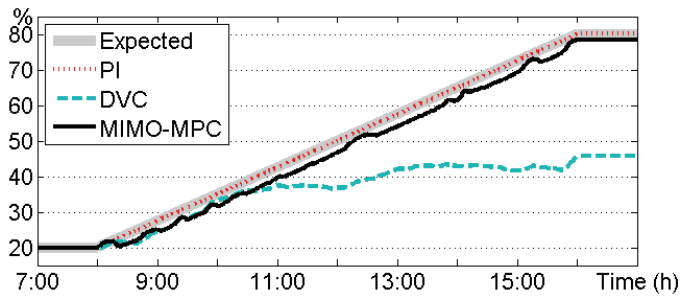

(b)

Fig. 9. Battery SOC of case 2: (a) area 1; (b) area 2.

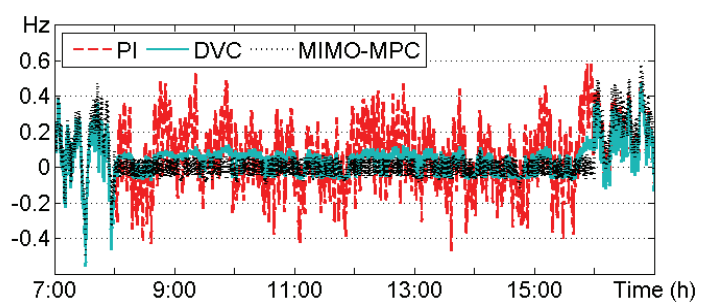

(a)

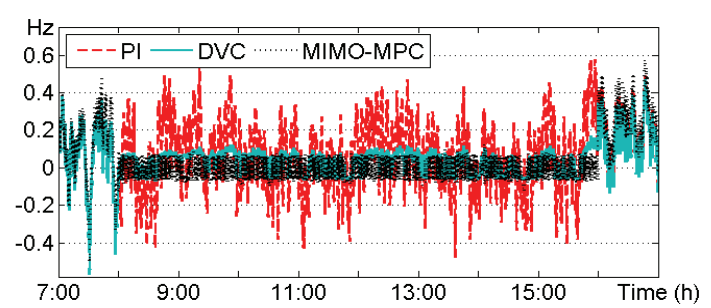

(b)

Fig. 10. Frequency deviation of case 2: (a) area 1; (b) area 2 
respectively. The MIMO-MPC and PI are able to obtain the desired SOC successfully, while the DVC is not able to achieve the desired SOC. Figs. 10 (a) and (b), show the frequency deviation of three methods in areas 1 and 2, respectively. The frequency deviations in case of MIMOMPC and DVC are lower than that of PI.

For case 3, EVs are assumed to be integrated into both areas. The battery SOC of the PI, DVC, and MIMO-MPC in areas 1 and 2, are shown in Figs. 11 (a) and (b), respectively. It can be seen that the DVC is not able to obtain the desired SOC in area 2, while the PI and MIMOMPC are able to produce the desired SOC for both areas successfully. Figs. 12 (a) and (b), show the frequency deviation of three methods in areas 1 and 2, respectively. As mentioned before, the specification of frequency deviation of MIMO-MPC and DVC controllers are defined at $\pm 0.05 \mathrm{~Hz}$. The frequency deviations of MIMO-MPC

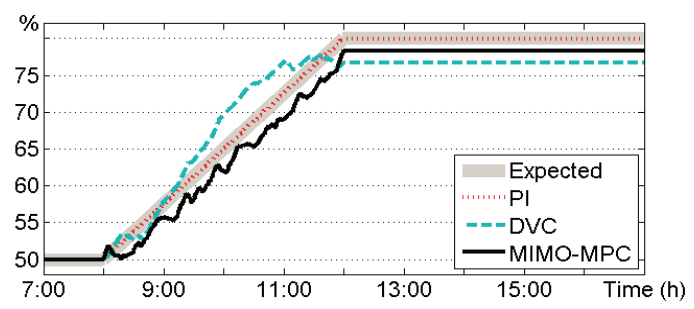

(a)

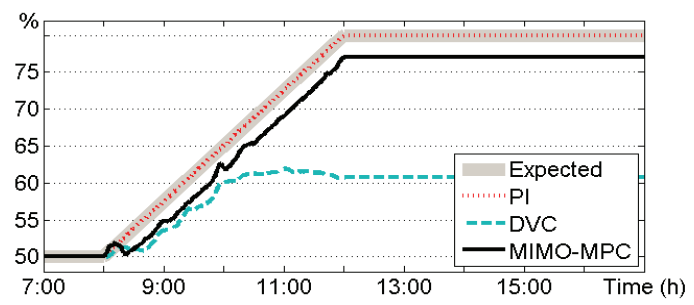

(b)

Fig. 11. Battery SOC of case 3: (a) area 1; (b) area 2

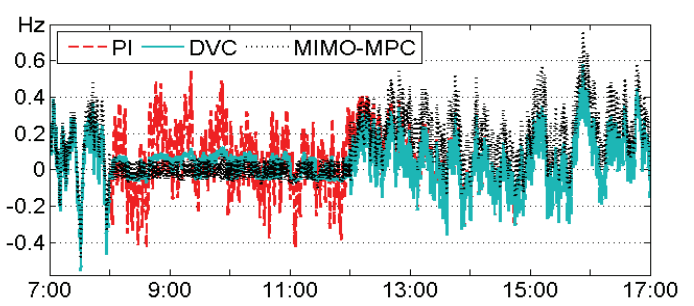

(a)

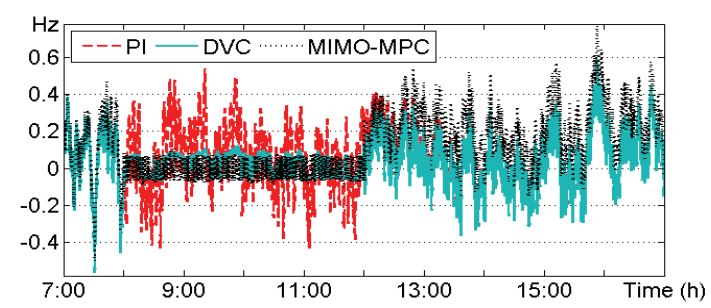

(b)

Fig. 12. Frequency deviation of case 3: (a) area 1; (b) area 2 and DVC are varied in the specified range of $\pm 0.05 \mathrm{~Hz}$. Clearly, the frequency deviation in case of MIMO-MPC and DVC are lower than that of the PI.

For case 4, EVs are assumed to be integrated into both areas. The battery SOC of PI, DVC, and MIMO-MPC controllers in areas 1 and 2, are shown in Figs. 13 (a) and (b), respectively. Figs. 14 (a) and (b), show the frequency deviation of PI, DVC and MIMO-MPC in areas 1 and 2, respectively. The frequency deviation in case of DVC and MIMO-MPC are lower than that of PI. Figs. 15 (a) and (b) show the EV power in areas 1 and 2, respectively. Figs. 16 (a) and (b) show the thermal power in areas 1 and 2, respectively.

As shown in Figs. 13 and 16, for the MIMO-MPC method, when the SOC ramp rate of both areas change (see Fig. 13, 8:00 h-12:00 h), the thermal output power in area 1 increases to $0.70 \mathrm{pu}$ while that in area 2 decreases to 0.11

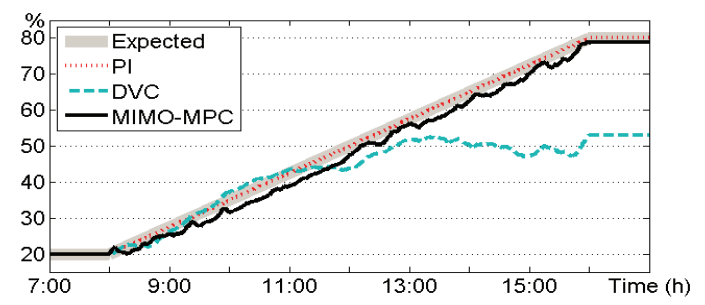

(a)

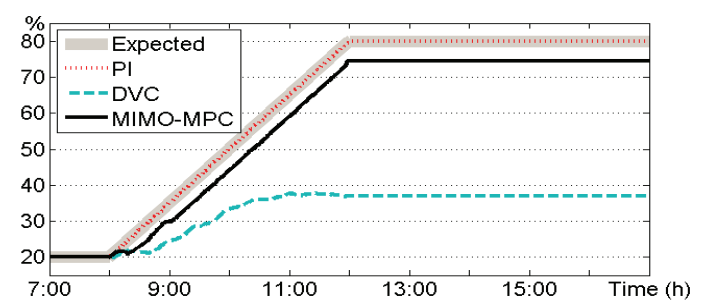

(b)

Fig. 13. Battery SOC of case 4: (a) area 1; (b) area 2

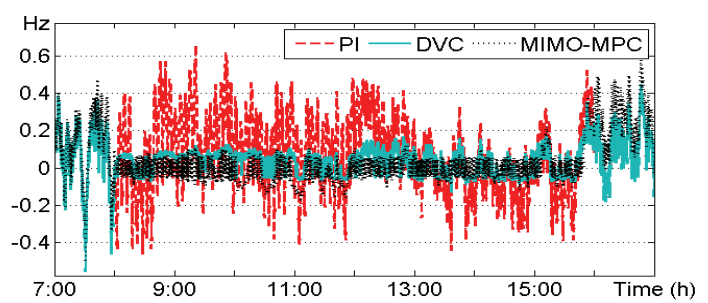

(a)

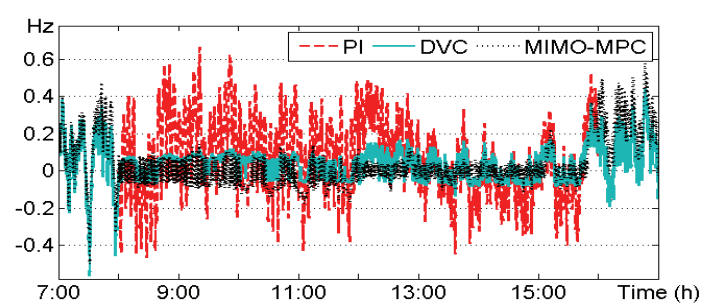

(b)

Fig. 14. Frequency deviation of case 4: (a) area 1; (b) area 2 


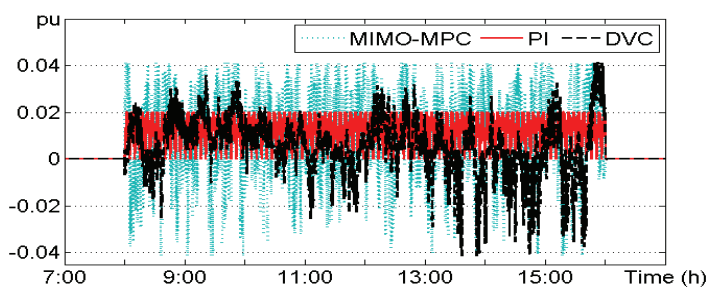

(a)

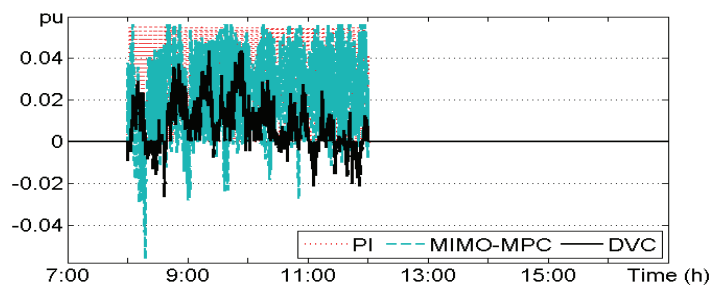

(b)

Fig. 15. EV power of case 4: (a) area 1; (b) area 2.

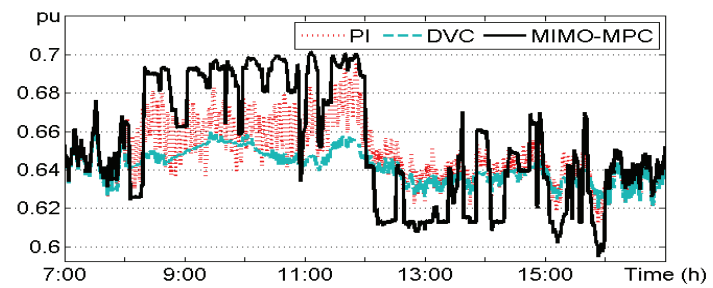

(a)

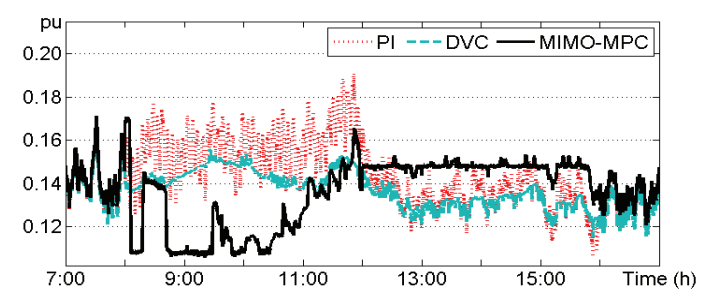

(b)

Fig. 16. Thermal power of case 4: (a) area 1; (b) area 2

pu. Besides, when the SOC ramp rate of area 1 changes, and that of area 2 does not change (12:00 h-16:00 h), the thermal output power in area 1 decreases to $0.60 \mathrm{pu}$. These results imply that the thermal output power in areas 1 and 2 depend on the SOC ramp rate in both areas.

As shown in Figs. 8-16, it can be seen that the proposed method can't control the frequency deviations after the SOC reaches the objective value. To solve this problem, the SOC objective function is changed to support frequency stabilization for all time of simulation. Therefore, the $S O C_{\mathrm{e}}$ in (2) is modified as follows,

$$
S O C_{e}(t)= \begin{cases}S O C_{\text {in }}, & T_{\text {in }} \leq t<T_{\text {in,charge }} \\ S O C_{\text {in }}+\frac{S O C_{\text {out }}-S O C_{\text {in }}}{T_{\text {out,charge }}-T_{\text {in,charge }}}, & T_{\text {in,charge }} \leq t \leq T_{\text {out,charge }}(7) \\ S O C_{\text {out }}, & T_{\text {out,charge }}<t \leq T_{\text {out }},\end{cases}
$$

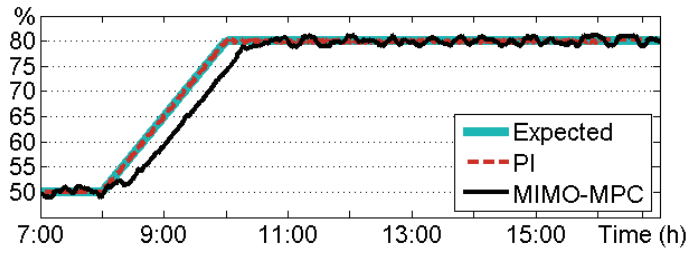

(a)

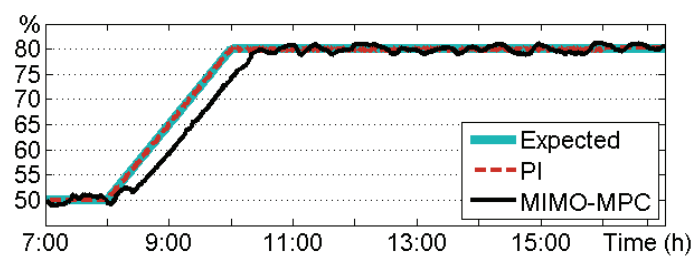

(b)

Fig. 17. Battery SOC of case 3 when change SOC objective function: (a) area 1; (b) area 2.

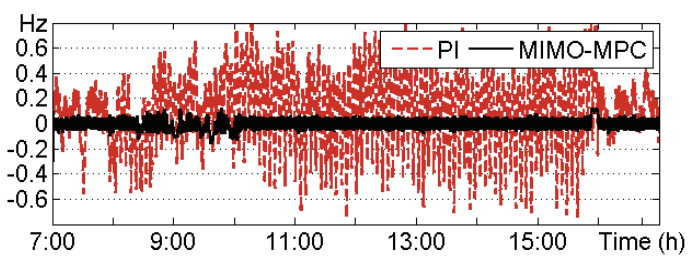

(a)

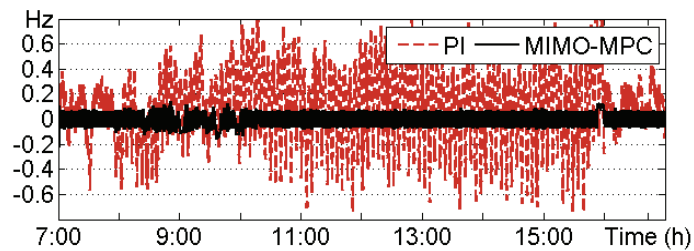

(b)

Fig. 18. Frequency deviation of case 3 when change SOC objective function: (a) area 1; (b) area 2

where $T_{\text {in }}$ and $T_{\text {out }}$ are the time for PHEV begin, and finish participated as $\mathrm{V} 2 \mathrm{G}$ for supporting frequency stabilization, respectively, $T_{i n \text {,charge }}$ and $T_{\text {out,charge }}$ are the time for PHEV begin, and finish charging to produce higher SOC as well as to support frequency stabilization, respectively.

Figs. 17 and 18 show battery SOC and frequency deviation of case 3 when the SOC objective function is changed to support frequency stabilization for all time of simulation. Here, time duration used to charge battery is reduced to 2 hour in order to show the capability of (7) when the SOC objective function hold constant for long time (10:00-17:00). It can be seen that the MIMO-MPC is able to control frequency deviation when the SOC reach the desired objective value.

Figs. 19 and 20 show absolute maximum frequency deviation and absolute SOC deviation, when time duration for charging the initial SOC to the desired SOC is changed, respectively. It can be seen that when time duration for charging increases, the absolute maximum frequency 


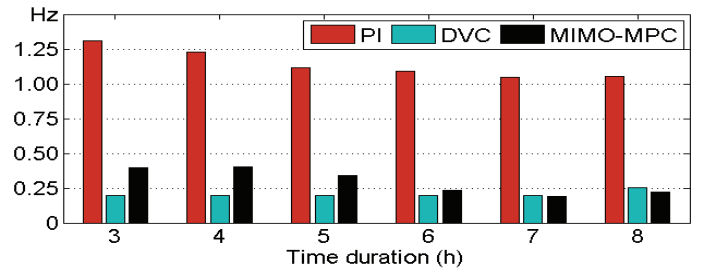

(a)

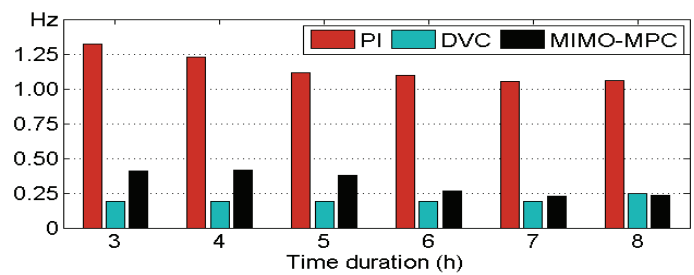

(b)

Fig. 19. Absolute maximum frequency deviation when time duration is changed for the $\mathrm{SOC}=20 \%$ to $80 \%$ : (a) area 1 ; (b) area 2

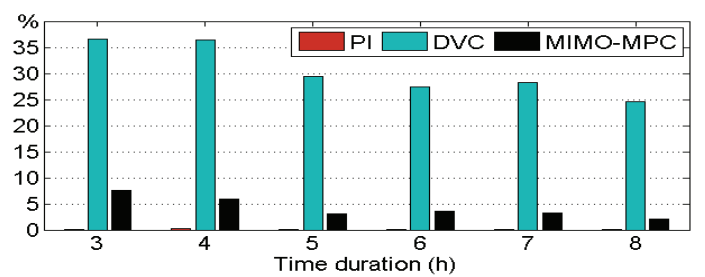

(a)

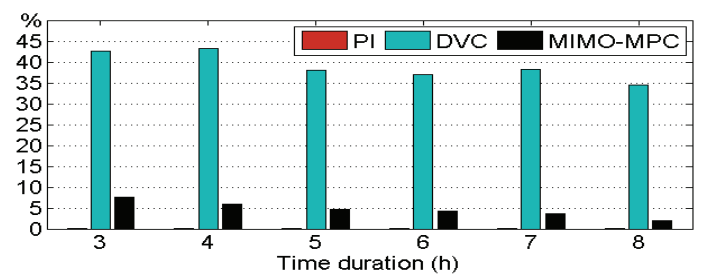

(b)

Fig. 20. Absolute SOC deviation when time duration is changed for the SOC $=20 \%$ to $80 \%$ : (a) area 1 ; (b) area 2

deviation in case of DVC and MIMO-MPC decrease and lower than that of PI method. Besides, when time duration for charging increases, the absolute SOC deviation in case of PI near zero while the DVC and MIMO-MPC are higher than the PI controller. However, the SOC deviation in case of the proposed MIMO-MPC method is lower than that of the DVC. Clearly, absolute maximum frequency deviation and absolute SOC deviation of the proposed MIMO-MPC method are lower than those of the PI and DVC for both areas.

In addition, as shown in Figs. 7 and 20, it can be seen that random load pattern of area 1 is changed more than area 2, wind power of area 1 is varied more than area 2, and SOC deviation of the DVC method of area 1 lower than of area 2. These details imply that the SOC deviation of DVC method is changed when random load pattern and wind power are changed. However, SOC deviation of the MIMO-MPC is not different for both areas. These results imply that the proposed MIMO-MPC method is robust to random load and wind power.

\section{Conclusion}

In this paper, the MIMO-MPC is applied for coordinating control of the LFC and EV in order to obtain the desired SOC as well as to stabilize the frequency fluctuation in the smart grid. By the MIMO-MPC optimal control signal calculated at each instant time based on the desired battery SOC and frequency constrains, the proposed controller is able to reduce the frequency fluctuation and provide the desired EV battery SOC at each instant time, concurrently. Simulation results on the twoarea inter-connected power system with the large wind power penetration, confirmed that the proposed MIMOMPC is able to reduce the frequency fluctuation and obtain battery SOC at the certain time over PI and DVC controllers.

\section{Acknowledgements}

This work was supported by the Office of the Higher Education Commission and the Thailand Research Fund, under Grant number MRG5980229.

\section{References}

[1] J. Gallardo-Lozano, M. I. Milanes-Montero, M. A. Guerrero-Martínez, E. Romero-Cadaval, "Electric vehicle battery charger for smart grids," Electr. Pow. Syst. Res., vol. 90, pp. 18-29, 2012.

[2] C.-T. Li, C. Ahn, H. Peng, and J. Sun, "Synergistic control of plug-in vehicle charging and wind power scheduling," IEEE Trans. Power Syst., vol. 28(2), pp. 1113-1121, 2013.

[3] M. Esmaili, and A. Goldoust, "Multi-objective optimal charging of plug-in electric vehicles in unbalanced distribution networks," Int. J. Electr. Power Energy Syst., vol. 73, pp. 644-652, 2015.

[4] M. A. López, S. de la Torre, S. Martín, and J. A. Aguado, "Demand-side management in smart grid operation considering electric vehicles load shifting and vehicle-to-grid support," Int. J. Electr. Power Energy Syst., vol. 64, pp. 689-698, 2015.

[5] X. Lin, J. Sun, S. Ai, X. Xiong, Y. Wanc, and D. Yang, "Distribution network planning integrating charging stations of electric vehicle with V2G," Int. J. Electr. Power Energy Syst., vol. 63, pp. 507-512, 2014.

[6] C. Rathore, and R. Roy, "Impact of wind uncertainty, plug-in-electric vehicles and demand response 
program on transmission network expansion planning," Int. J. Electr. Power Energy Syst., vol. 75, pp. 59-73, 2016.

[7] P. Mitra, and G.K. Venayagamoorthy, "Wide area control for improving stability of a power system with plug-in electric vehicles," IET Gener. Distrib., vol. 4(10), pp. 1151-1163, 2009.

[8] Y. Ota, H. Taniguchi, T. Nakajima, K. M. Liyanage, J. Baba, and A. Yokoyama, "Autonomous distributed V2G (vehicle-to-grid) considering charging request and battery condition," in Proc. IEEE PES Innov. Smart Grid Technol. Conf. Eur., Oct. 2010, pp. 1-6.

[9] Y. Ota, H. Taniguchi, T. Nakajima, K.M. Liyanage, J. Baba, and A. Yokoyama, "Autonomous distributed V2G (vehicle-to-grid) satisfying scheduled charging," IEEE Trans. Smart Grid, vol. 3(1), pp. 559-564, 2012.

[10] H. Liu, Z. Hu, Y. Song, and J. Lin, "Decentralized vehicle-to-grid control for primary frequency regulation considering charging demands," IEEE Trans. Power Syst., vol. 28(3), pp. 3480-3489, 2013.

[11] H. Liu, Z. Hu, Y. Song, J. Wang, and X. Xie, "Vehicle-to-grid control for supplementary frequency regulation considering charging demands, IEEE Trans. Power Syst., to be published.

[12] J. Pahasa and I. Ngamroo, "PHEVs bidirectional charging/discharging and SOC control for microgrid frequency stabilization using multiple MPC," IEEE Trans. Smart Grid, vol. 6(2), pp. 526-533, 2015.

[13] T. Masuta and A. Yokoyama, "Supplementary load frequency control by use of a number of both electric vehicles and heat pump water heaters, IEEE Trans. Smart Grid, vol. 3(3), pp. 1253-1262, 2012.

[14] S. Vachirasricirikul and I. Ngamroo, "Robust LFC in a smart grid with wind power penetration by coordinated V2G control and frequency controller," IEEE Trans. Smart Grid, vol. 5(1), pp. 371-380, 2014.

[15] D.E. Seborg, T.F Edgar, D.A. Mellichamp, "Process Dynamics and Control," $2^{\text {nd }}$ Edition, John Wiley \& Sons, Inc., 2003.

[16] D. Lauri, J. V. Salcedo, S. Garci-Nieto, and M. Martinez, "Model predictive control relevant identification: multiple input multiple output against multiple input single output," IET Control Theory Appl., vol. 4(9), pp. 1756-1766, 2010.

[17] M. Morari, and N.L. Ricker, Model Predictive Control Toolbox, MATH WORKS Inc., 1998.

[18] A. Bemporad, M. Morari, and N.L. Ricker, Model Predictive Control Toolbox ${ }^{\mathrm{TM}}$ User's Guide, MATH WORKS Inc., 2013.

[19] T.H. Mohamed, J. Morel, H. Bevrani, and T. Hiyama, "Model predictive based load frequency control design concerning wind turbines," Int. J. Electr. Power Energy Syst., vol. 43, pp. 859-867, 2012.

[20] M. Maa, H. Chen, X. Liu, and F. Allgöwer, "Distributed model predictive load frequency control of multi-area interconnected power system," Int. J.
Electr. Power Energy Syst., vol. 62, pp. 289-298, 2014.

[21] Md. Parvez Akter, Saad Mekhilef, Nadia Mei Lin Tan and Hirofumi Akagi, "Model predictive control of bidirectional AC-DC converter for energy storage system" J. Electr. Eng. Technol., vol. 10(1), pp. 165$175,2015$.

[22] J. Kennedy and R. Eberhart, "Particle swarm optimization," in Proc. IEEE Int. Conf. Neural Net., vol. 4, pp. 1942-1948, 1995.

[23] T. Inoue and H. Amano, "A Thermal power plant model for dynamic simulation of load frequency control, Power Systems Conf. and Exp. 2006, PSCE '06. 2006 IEEE PES," Oct. 29 -Nov. 1, 2006, Atlanta, GA.

[24] I. Ngamroo, "Robust decentralized frequency stabilizers design for SMES taking into consideration system uncertainties," Electric Power Syst. Research, vol. 74, pp. 281-292, 2005.

[25] J. Pahasa and I. Ngamroo, "Coordinated control of wind turbine blade pitch angle and PHEVs using MPCs for load frequency control of microgrid," IEEE Syst. J, vol. 10(1), pp. 97-105, 2016.

[26] M. Soliman, O.P. Malik, and D.T. Westwick, "Multiple model MIMO predictive control for variable speed variable pitch wind turbines, " 2010 American Control Conf., Marriott Waterfront, Baltimore, MD, USA, June 30-July 02, 2010.

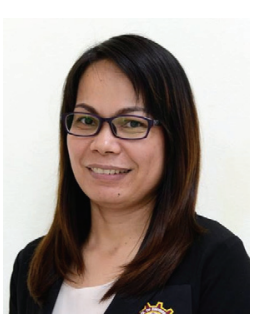

Jonglak Pahasa received the B.Eng. degree from the King Mongkut's Institute of Technology Ladkrabang (KMITL), Bangkok, Thailand; the M.Eng. degree from Chiang Mai University, Chiang Mai, Thailand; and the D.Eng. degree from KMITL, in 1997, 2007, and 2011, respectively, all in electrical engineering. She is currently an Assistant Professor with the School of Engineering, University of Phayao, Phayao, Thailand. Her current research interests include the applications of artificial intelligence in power system stability and control.

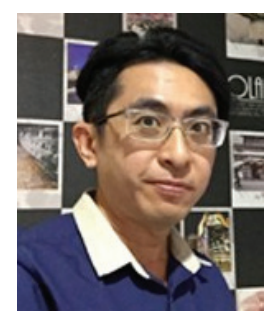

Issarachai Ngamroo received the Ph.D. degree in Electrical Engineering from Osaka University, Japan, in 2000. Currently, he is a Professor with the Department of Electrical Engineering, Faculty of Engineering, King Mongkut's Institute of Technology Ladkrabang, Bangkok, Thailand. His research interests include power system stability, dynamic, and control. Prof. Ngamroo is a senior member of the IEEE. 